\title{
A Role for Glycosphingolipid Accumulation in the Renal Hypertrophy of Streptozotocin-induced Diabetes Mellitus
}

\author{
Ivan Z. Zador, * Gayatri D. Deshmukh, ${ }^{*}$ Robin Kunkel, ${ }^{3}$ Kent Johnson, " Norman S. Radin," and James A. Shayman* \\ Departments of * Pediatrics, ${ }^{\ddagger}$ Internal Medicine, ${ }^{\S}$ Pathology, and $"$ Mental Health Research Institute, \\ University of Michigan Medical Center, Ann Arbor, Michigan 48109
}

\begin{abstract}
Glucosylceramide (GlcCer) and related glycosphingolipids have been implicated as causal elements in both the growth of cells and in the regulation of hormonal signaling. We therefore studied whether the renal hypertrophy induced by diabetes was associated with enhanced synthesis of glycosphingolipids. $16 \mathrm{~d}$ after the induction of diabetes, increases in renal size and concentration of glucocerebroside and ganglioside GM3 were observed paralleling an increase in UDP-Glc concentration. GlcCer synthase and $\boldsymbol{\beta}$-glucosidase-specific activities were no different between control and diabetic kidneys. The apparent $K_{m}$ of the GlcCer synthase with respect to UDP-Glc was 250 $\mu \mathrm{M}$ and was unchanged in the diabetic kidneys. The observed concentrations of UDP-Glc were 149 and $237 \mu \mathrm{M}$ in control and diabetic kidneys, respectively. The UDP-Glc level is thus rate limiting with regard to GlcCer synthesis. To determine whether the changes in glycolipid content were functionally significant, diabetic and control groups were treated with the GlcCer synthase inhibitor, D-threo-1-phenyl-2-decanoylamino-3-morpholino-1-propanol, 2 wk after the induction of diabetes. Kidney weights in the diabetic rats treated with $D-$ threo-1-phenyl-2-decanoylamino-3-morpholino-1-propanol were no different than the control groups. Morphometric analysis of glomerular volumes paralleled changes in renal growth. Glycosphingolipid formation may therefore represent a significant pathway for glucose utilization in early diabetic nephropathy. (J. Clin. Invest. 1993. 91:797-803.) Key words: kidney • glycosphingolipids $\cdot$ ceramide $\bullet$ glucosylceramide $\bullet$ streptozotocin
\end{abstract}

\section{Introduction}

Under conditions of hyperglycemia, several normally minor pathways of glucose metabolism become more prominent. In the kidney these include the formation of sorbitol (1), the nonenzymatic glycosylation of proteins (2), and the de novo formation of fatty acylglycerides (3). These pathways are the basis for recent hypotheses on the mechanisms of diabetic complications, including nephropathy. However, no one hypothesis can account for the multitude of early and late changes associated with diabetic renal injury.

Address correspondence to James A. Shayman, Department of Internal Medicine, University of Michigan Medical Center, Ann Arbor, MI 48109-0676.

Received for publication 12 June 1992 and in revised form 7 October 1992.

J. Clin. Invest.

(C) The American Society for Clinical Investigation, Inc.

0021-9738/93/03/0797/07 \$2.00

Volume 91, March 1993, 797-803
Increases in UDP-hexose levels have been reported in the kidney and other organs in association with experimental diabetes $(4,5)$. These changes have been postulated to increase the expression of glycoproteins and glycosaminoglycans in the basement membranes of diabetic tissues. Glycosphingolipids (GSLs),$^{1}$ however, also require nucleotide-hexoses for their synthesis. One can therefore expect increases in GSLs in diabetes. These lipids are of potential interest because they have been implicated in the regulation of growth and a variety of critical cellular functions. The present study reports the occurrence of increases in GSL expression in association with renal hypertrophy in diabetic rats and correlates these changes with increases in the levels of GSL precursors.

\section{Methods}

Materials. GlcCer was isolated from the spleen of a patient with Gaucher disease (6). Ganglioside standards were from Matreya, Inc. (Pleasant Gap, PA). NBD-GlcCer, streptozotocin, UDP-Glc (from yeast ), UDP-Glc dehydrogenase (from bovine liver), and Na taurocholate were from Sigma Immunochemicals (St. Louis, MO). Ultralente Iletin I insulin was from Eli Lilly and Co. (Indianapolis, IN). SpragueDawley rats were from Harlan Industries (Indianapolis, IN).

Induction of diabetes. Male rats were fed a standard pellet laboratory chow and were provided with water ad lib. Diabetes was induced by the administration of streptozotocin ( $80 \mathrm{mg} / \mathrm{kg}$ i.p.) dissolved in 10 $\mathrm{mM} \mathrm{Na}$ citrate, $\mathrm{pH} 5.5$; controls were injected with buffer alone. Animals were provided with immediate access to food. Blood was obtained from the tail vein and blood glucose levels were determined with a glucose analyzer (Beckman Instruments, Inc., Fullerton, CA). Animals with blood glucose levels $>35 \mathrm{mM}$ were hypercatabolic and did not reliably exhibit renal hypertrophy. Therefore only animals with blood glucose concentrations between 13.5 and $32 \mathrm{mM}$ were used. At the end of the experimental period, the animals were weighed, anesthetized with ether, and their kidneys removed and weighed. For UDP-Glc measurements, the kidneys were frozen in liquid nitrogen immediately after removal.

Glycolipid analysis. Kidneys were homogenized in 10 vol of chloroform:methanol (1:1) with a Polytron (Brinkmann Instruments Co., Westbury, NY) and the homogenates were filtered through a sintered glass funnel. The pellet was extracted twice with $10 \mathrm{ml}$ of chloroform:methanol (1:2), and the pooled extracts were evaporated to dryness and redissolved in chloroform:methanol:water (30:60:8). The lipid extracts were analyzed as previously described (7-9).

TLC plates were sprayed with a cupric sulfate charring reagent (10) made by a recently described modification (11). Lipids were quantitated by densitometry by comparison to known amounts of authentic standards. Only the nonhydroxy GlcCer bands were measured.

UDP-Glc determination. UDP-Glc was assayed enzymatically by the method of Keppler et al. (12). In brief, frozen kidneys were weighed and homogenized in $5 \mathrm{vol}$ of $0.6 \mathrm{~N}$ perchloric acid. The homogenate was centrifuged at $1,000 \mathrm{~g}$ and $\mathrm{KHCO}_{3}$ was added to the

1. Abbreviations used in this paper: GSL, glycosphingolipid; PDMP, D-threo-1-2-decanoylamino-3-morpholino-1-propanol. 
supernatant to achieve a pH of 8.5 . The $\mathrm{KClO}_{4}$ was removed by centrifugation. A portion of the supernatant $(0.2-0.4 \mathrm{ml})$ was added to $0.5 \mathrm{ml}$ of glycine- $\mathrm{KOH}(0.5 \mathrm{M}, \mathrm{pH} 8.7)$ containing $3 \mathrm{mM} \mathrm{NAD}^{+}$and $6 \mathrm{mM}$ sodium EDTA. Water was added to yield a final vol of $1 \mathrm{ml}$. UDP-Glc dehydrogenase ( $10 \mu l, 0.09 \mathrm{U})$ was added and the ultraviolet absorbance was measured immediately and 10 min later.

Enzyme assays. The kidneys were stored at $-70^{\circ} \mathrm{C}$ and homogenized in 9 vol of water before assay. Aliquots of each homogenate were assayed in duplicate. Glucosylceramide $\beta$-glucosidase (EC 3.2.1.45) was assayed with the fluorogenic substrate, NBD-GlcCer, with $0.1-0.2$ $\mathrm{mg}$ of tissue in a total volume of $0.2 \mathrm{ml}$ (13). GlcCer from Gaucher spleen, Triton X-100, Na taurocholate, and phosphate-citrate buffer (pH 5.0) were employed.

Ceramide:UDP-Glc glucosyltransferase (EC 2.4.1.80) was assayed with an improved method using NAD to protect the nucleotide sugar against pyrophosphatase (14).

D-threo-1-phenyl-2-decanoylamino-3-morpholino-1-propanol (PDMP) treatment. PDMP was administered as the DL-hydrochloride to both diabetic and control animals $14 \mathrm{~d}$ after the induction of diabetes with streptozotocin. Previous studies have demonstrated that the circulating plasma levels of PDMP are prolonged in the presence of the cytochrome P450 inhibitor, piperonyl butoxide (15). The piperonyl butoxide was prepared by dissolution in corn oil at a concentration of $150 \mathrm{mg} / \mathrm{ml}$ oil. The butoxide solution was administered intraperitoneally before injection of PDMP. On the first day of treatment this was given $4 \mathrm{~h}$ before PDMP treatment; on days 2 to 4 of treatment it was administered just before the PDMP injection. PDMP was prepared as an emulsion with the detergent Myrj 52 in normal saline, buffered with sodium acetate. The final concentrations of PDMP, Myrj 52, and sodium acetate in $\mathrm{mg} / \mathrm{ml}$ were 6,12 , and 8 , respectively. The PDMP was given intraperitoneally as a dose of $100 \mathrm{mg} / \mathrm{kg}$ body wt.

Glomerular morphometry. Glomerular volumes were measured on paraffin sections of kidney fixed in formalin. 3- $\mu \mathrm{m}$ sections were stained with periodic acid Schiff reagent. The measurements required the determination of the mean glomerular random cross-sectional area $(A(G))$. Glomerular areas were measured using a camera (Chromachip; Javelin Electronics, Torrance, CA ) attached to an Olympus BH-2 microscope interfaced with a Macintosh II computer containing an Image Capture 2 frame grabber (Scion Corp., Walkerville, MD) and image 1.38B14 software (NIH Public Software, Bethesda, MD). Glomerular volumes were derived by the method of Weibel (16) as applied by Hirose et al. (7).

\section{Results}

In the first study, three groups of male rats weighing an average of $113 \mathrm{~g}$ ( range $105-124 \mathrm{~g}$ ) were made diabetic with streptozotocin. After $24 \mathrm{~h}$, one group of rats was treated with daily injections of Ultralente Iletin insulin at an initial dose of $0.03 \mathrm{U} / \mathrm{g}$

Table I. Characteristics of Control, Diabetic, and Insulin-treated Rats

\begin{tabular}{lcccc}
\hline & Body wt & Kidney wt & $\begin{array}{c}\text { Kidney } \\
\text { wt/body wt }\end{array}$ & $\begin{array}{c}\text { Blood } \\
\text { glucose }\end{array}$ \\
\hline & $g$ & $g$ & $g / 100 g$ & $m M$ \\
Control & $280 \pm 7.2$ & $2.73 \pm 0.13$ & 0.98 & $9.1 \pm 0.13$ \\
Diabetic & $204 \pm 8.2$ & $3.28 \pm 0.16$ & 1.60 & $21.1 \pm 1.36$ (day 2) \\
& & & & $22.6 \pm 1.21$ (day 15) \\
Insulin-treated & $284 \pm 4.0$ & $2.78 \pm 0.11$ & 0.96 & $26.3 \pm 3.12$ (day 2) \\
& & & & $6.6 \pm 1.22$ (day 15)
\end{tabular}

The data are expressed as the mean $\pm \mathrm{SD}$ for six animals per group.
Table II. Glycosphingolipid and UDP-Glc Levels in Diabetic and Insulin-treated Diabetic Rats

\begin{tabular}{lccr}
\hline & Control & Diabetic & Insulin-treated \\
\hline GlcCer & $29.4 \pm 0.91$ & $37.8 \pm 1.42^{*}$ & $33.5 \pm 1.21$ \\
Ganglioside GM3 & $43.9 \pm 3.13$ & $65.4 \pm 6.46^{*}$ & $44.3 \pm 12.5$ \\
UDP-Glc & $78.4 \pm 4.1$ & $125 \pm 7.3^{*}$ & $105 \pm 8.14$ \\
& & & \\
\hline
\end{tabular}

UDP-Glc levels are expressed as nmol/g wet wt of kidney. The data for GlcCer and ganglioside GM3 are expressed as $\mu \mathrm{g} / \mathrm{g}$ wet wt. The GlcCer data represent densitometric measurements from the fastest moving cerebroside spot (representing cerebrosides not containing phytosphingosine or 2-hydroxy fatty acids), although all spots migrating as cerebrosides increased visibly in the diabetic kidneys. ${ }^{*} P$ $<0.05$ by analysis of variance and the Scheffe $F$ test. The data are expressed as the mean $\pm \operatorname{SE}(n=6)$.

body wt. Daily blood glucose determinations were made and the insulin dose adjusted for each rat to approach euglycemia. Initially the rats responded poorly to the insulin (Table I), exhibiting a mean blood glucose of $15.9 \pm 0.7 \mathrm{mM}$ during days 2 through 12. However, their glucose levels normalized toward the end of the experimental period.

In spite of an increased caloric intake, the untreated diabetic rats weighed significantly less $(27 \%)$ than the control rats
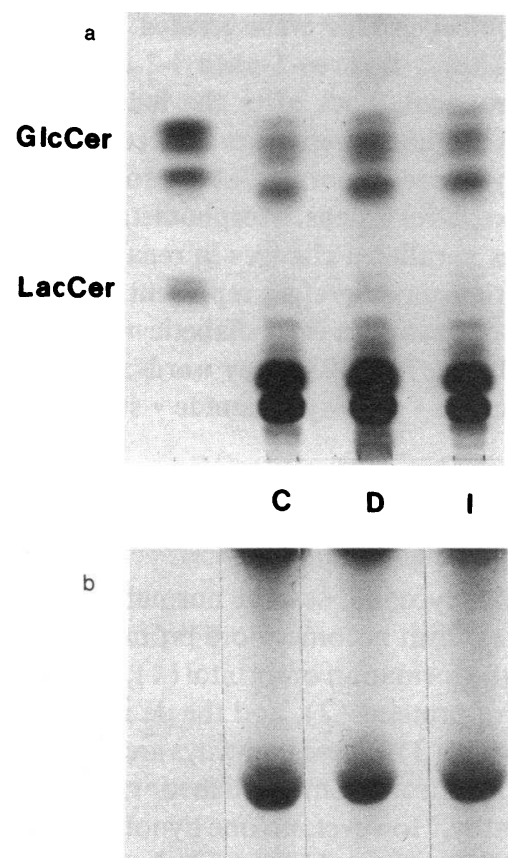

Figure 1. Effect of diabetes on $(a)$ neutral and (b) acidic GSL content of rat kidneys. Control $(C)$, diabetic $(D)$, or

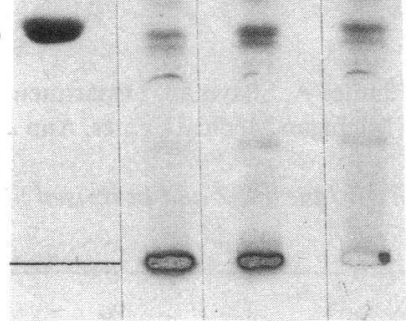
insulin-treated diabetic (I) rats were studied 16 $\mathrm{d}$ after the induction of diabetes with streptozotocin. Lipids were separated by TLC and detected by charring. GlcCer, glucosylceramide; LacCer, lactosylceramide; GM3, ganglioside GM3. 
Table III. GlcCer Metabolizing Enzyme Activities from Control and Diabetic Rats

\begin{tabular}{lcc}
\hline & Control & Diabetic \\
\hline$\beta$-glucosidase & $211 \pm 13.5$ & $200 \pm 13.5$ \\
GlcCer synthase & $58 \pm 1.6$ & $59 \pm 1.3$ \\
\hline
\end{tabular}

Glucosylceramide $\beta$-glucosidase activity is expressed as $\mathrm{nmol} / \mathrm{mg}$ tissue per $\mathrm{h} \pm \mathrm{SE}$; GlcCer synthase activity is expressed as $\mathrm{pmol} / \mathrm{mg}$ tissue per $\mathrm{h} \pm \mathrm{SE}$. $\beta$-glucosidase and GlcCer synthase assays used 0.2 $\mathrm{mg}$ and $2.0 \mathrm{mg}$ of kidney, respectively. Values were not significantly different by paired $t$ test, $n=6$.

by the end of the experimental period. The insulin-treated rats, however, maintained the same body weight as the control rats. The kidney weights of the untreated diabetic rats were $20 \%$ larger than those of the control and insulin-treated groups. When normalized for body weight the increase was even more pronounced (63\%).

Glycosphingolipid and UDP-Glc measurements were performed on the kidneys from these animals (Table II and Fig. 1). Significant, large increases in both GlcCer $(29 \%)$ and ganglioside GM3 concentrations ( $49 \%$ ) were observed in the diabetic animals. Insulin-treated rats had renal GlcCer levels that were slightly higher than control but significantly lower than the diabetic group. The increase in ganglioside GM3 observed in the diabetic rats also was corrected by insulin treatment. UDPGlc levels were significantly higher $(59 \%)$ in the untreated diabetic animals and were partially, but significantly, normalized by the insulin treatment. Water comprises $\sim 76 \%$ of the wet wt of the kidneys (17). Assuming that one-third of the water was in extracellular compartments and that all of the UDP-Glc is intracellular, the estimated intracellular tissue concentrations of UDP-Glc would be 149,237 , and $199 \mu \mathrm{M}$ in control, diabetic, and insulin-treated kidneys, respectively.

To ascertain the basis for the increase in cerebroside levels in the untreated diabetic animals, GlcCer $\beta$-glucosidase and GlcCer synthase activities were measured ( Table III). Neither activity was different when compared to the control group. These data are consistent with the interpretation that increased cerebroside levels were likely caused by an increase in the UDP-Glc level. To further support this interpretation, the apparent $K_{\mathrm{m}}$ for UDP-Glc of the rat renal GlcCer synthase was measured. The apparent $K_{\mathrm{m}}$ was $250 \mu \mathrm{M}$ in both normal and diabetic kidney (Fig. 2). This concentration is of the order of or significantly greater than that calculated based on the observed

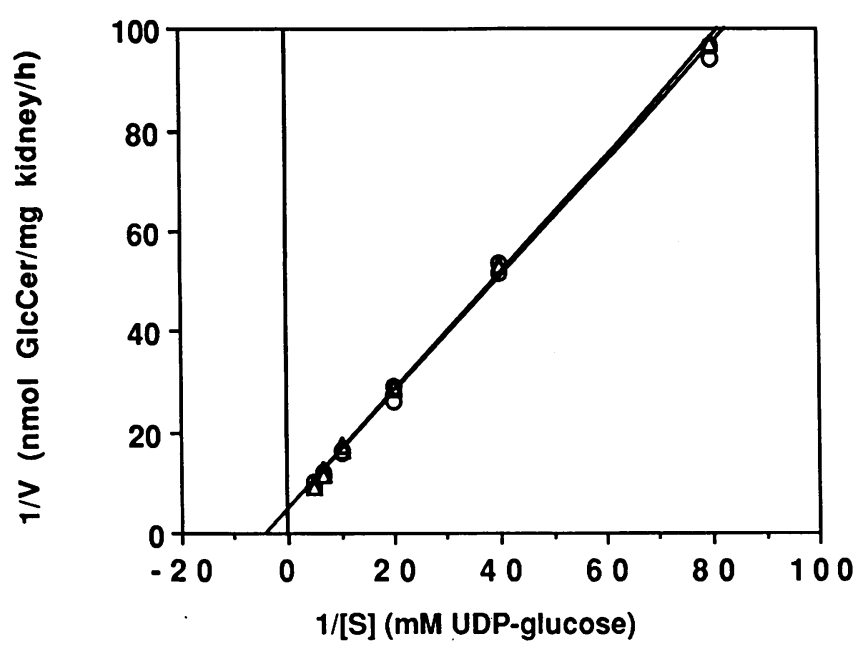

Figure 2. Kinetic analysis of the GlcCer synthase of diabetic and control kidneys. A Lineweaver-Burke plot based on the velocity $1 /(\mathrm{nmol} \mathrm{GlcCer} / \mathrm{mg}$ protein per $\mathrm{h})$ of the GlcCer synthesis vs. the concentration of substrate $1 /(\mathrm{mM}$ UDP-Glc $)$ of diabetic $(\Delta)$ and control (O).

contents of UDP-Glc. Since glucosylceramide synthase is topologically localized to the cytosolic side of the Golgi membrane $(18,19)$, the intracellular distribution of renal UDP-Glc was measured. Kidneys homogenized immediately after resection were centrifuged at $263,000 \mathrm{~g}$ for $10 \mathrm{~min}$ at $4^{\circ} \mathrm{C}$. Under these conditions, $>94 \%$ of the UDP-Glc remained in the cytosolic fraction and $<6 \%$ remained in the unwashed particulate fraction (data not shown). Thus the measured UDP-Glc concentrations were substantially that of the UDP-Glc in contact with the synthase.

A second study was performed to determine whether the increase in glucolipid formation seen in diabetes was functionally important. In this study one group of animals was made diabetic with streptozotocin, and the other group received vehicle alone. After $2 \mathrm{wk}$, the control and diabetic groups were each divided into two groups of similar mean weights. Half of the nondiabetic and half of the diabetic animals received piperonyl butoxide and DL-threo-PDMP. The control nondiabetic and diabetic groups were pair fed and received vehicle alone. After $4 \mathrm{~d}$ of treatment the animals were weighed and killed. The characteristics of these animals are shown in Table IV. The body weights of the PDMP-treated and untreated nondiabetic animals were not significantly different. The weights of the diabetic rats were significantly lower than the nondiabetic rats.

Table IV. Characteristics of Control and Diabetic Rats Treated with PDMP

\begin{tabular}{lcccccc}
\hline \multicolumn{1}{c}{ Group } & $n$ & Body wt & Kidney wt & Glucose & BUN & Creatinine \\
\hline & & $g$ & $g$ & $m M$ & $m g / d l$ & $m g / d l$ \\
Control & 12 & $241 \pm 16$ & $2.30 \pm 0.17$ & $10.1 \pm 0.83$ & $15 \pm 2.4$ & $0.28 \pm 0.07$ \\
Control + PDMP & 11 & $238 \pm 21$ & $2.25 \pm 0.27$ & $9.38 \pm 0.89$ & $13 \pm 3.2$ & $0.31 \pm 0.09$ \\
Diabetic & 7 & $203 \pm 23$ & $2.62 \pm 0.29^{*}$ & $25.9 \pm 3.22$ & $24 \pm 5.7$ & $0.23 \pm 0.05$ \\
Diabetic + PDMP & 7 & $200 \pm 11$ & $2.32 \pm 0.19$ & $25.9 \pm 4.33$ & $28 \pm 6.4$ \\
\end{tabular}

The data are expressed as the mean \pm SD. All animals received the cytochrome P450 inhibitor piperonyl butoxide $(600 \mathrm{mg} / \mathrm{kg}) \mathrm{intraperitoneally}$. Treated control and diabetic animals received $\mathrm{D}$, L-threo-PDMP. ${ }^{*} P<0.001$ by one-way analysis of variance using the Scheffe test. 

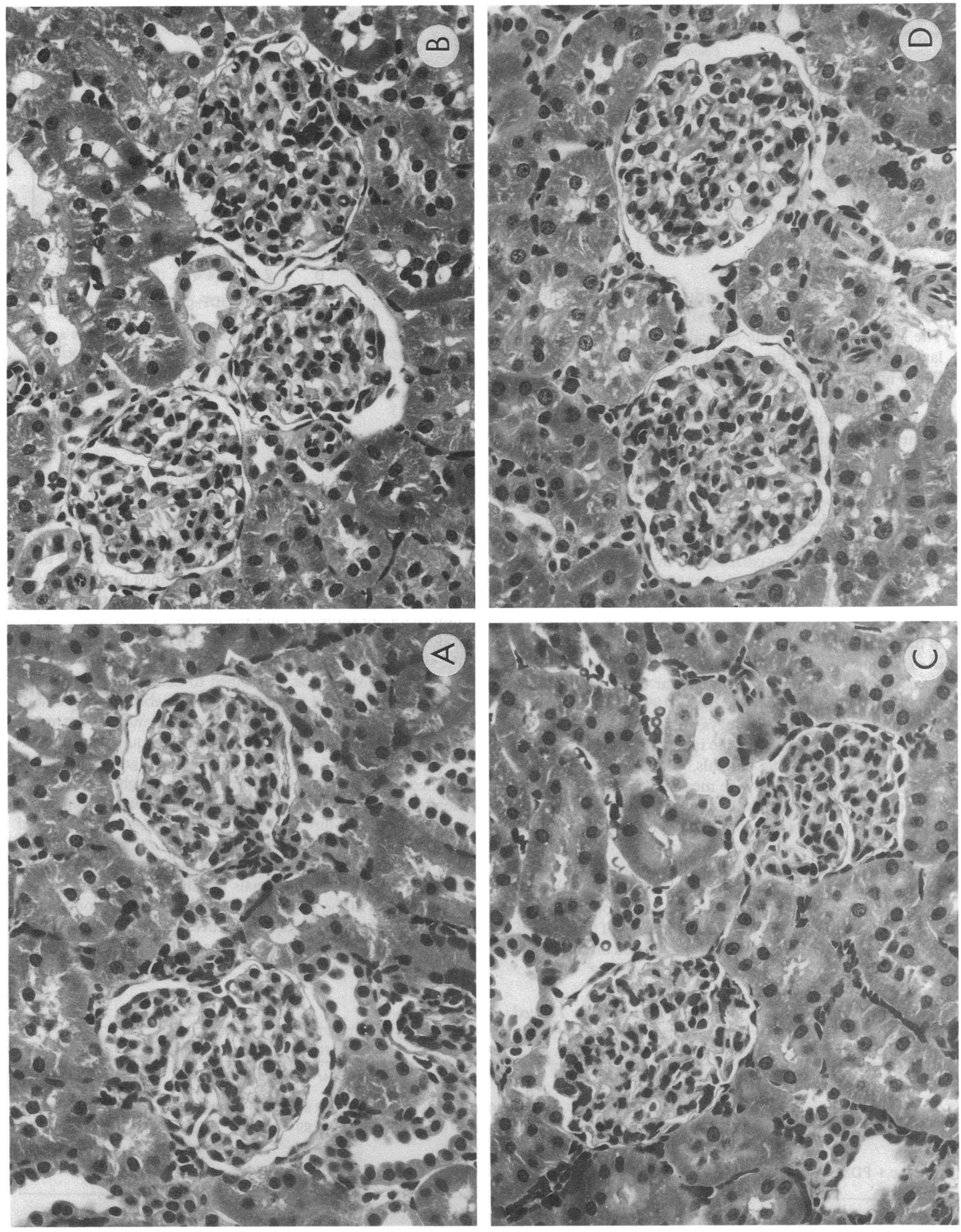


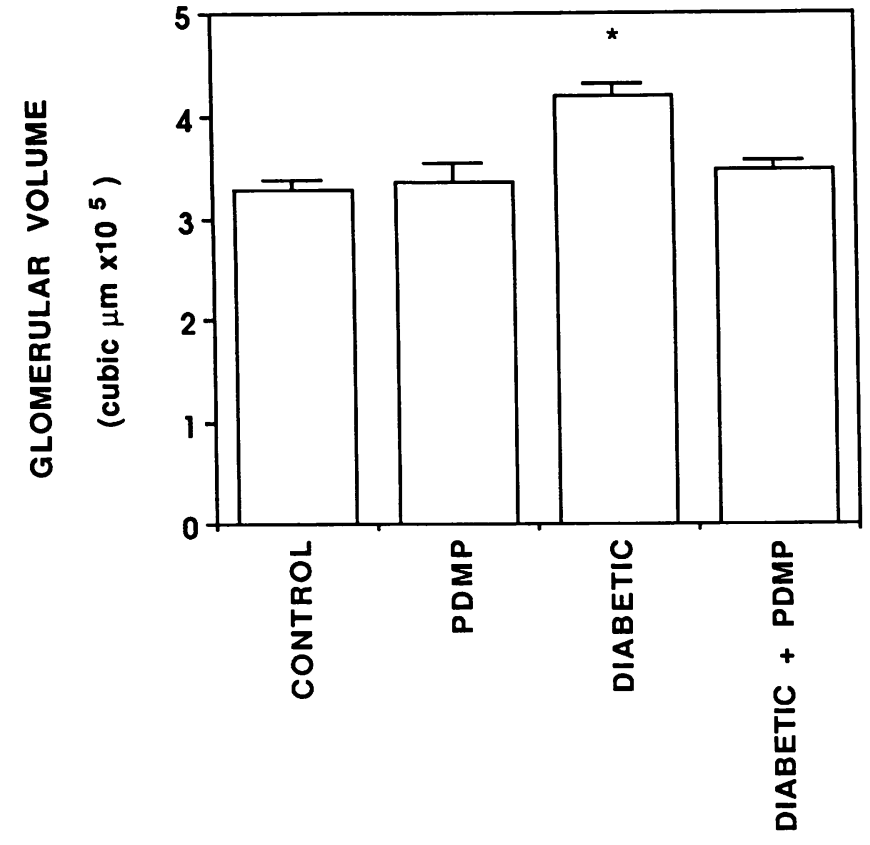

Figure 4. Glomerular volumes of control diabetic and PDMP-treated rats. Calculated glomerular volumes from control, PDMP-treated, diabetic, and inhibitor-treated diabetic animals. Morphometry was performed as described in Methods. ${ }^{*} P<0.001$ by one-way analysis of variance using the Scheffe test.

Moreover, there was no difference between PDMP-treated and untreated diabetic animals. Kidney weights, however, did differ significantly. The diabetic animals displayed kidney weights that were significantly greater than the control animals. The kidney weights of the diabetic animals treated with PDMP, however, were significantly lower than those of the untreated diabetic animals in spite of the fact that these animals were significantly hyperglycemic.

The serum creatinines of the diabetic animals were lower than those of the control groups, probably reflective of the lower body weights. The blood urea nitrogen levels were higher among the diabetic animals, consistent with the significant level of hyperglycemia.

Renal histology was performed to ascertain if the drug treatment induced significant toxicity (Fig. 3). Both periodic acid Schiff and hematoxylin and eosin-stained sections of kidney revealed no evidence of acute tubular necrosis, acute or chronic inflammation, or glomerular pathology. There was an apparent increase in the size of the glomeruli of the diabetic animals. This increase, however, was not obvious in the inhibitor treated diabetic animals.

In an attempt to quantify changes in glomerular size, five kidneys from each group of animals were studied by morphometric analysis (Fig. 4). Glomerular areas were determined on a randomly sectioned kidney from each group. Every available glomerulus from each section was measured with 237 to 357 glomeruli per experimental group. The mean glomerular volume of diabetic kidneys was $28 \%$ greater than that of the con-

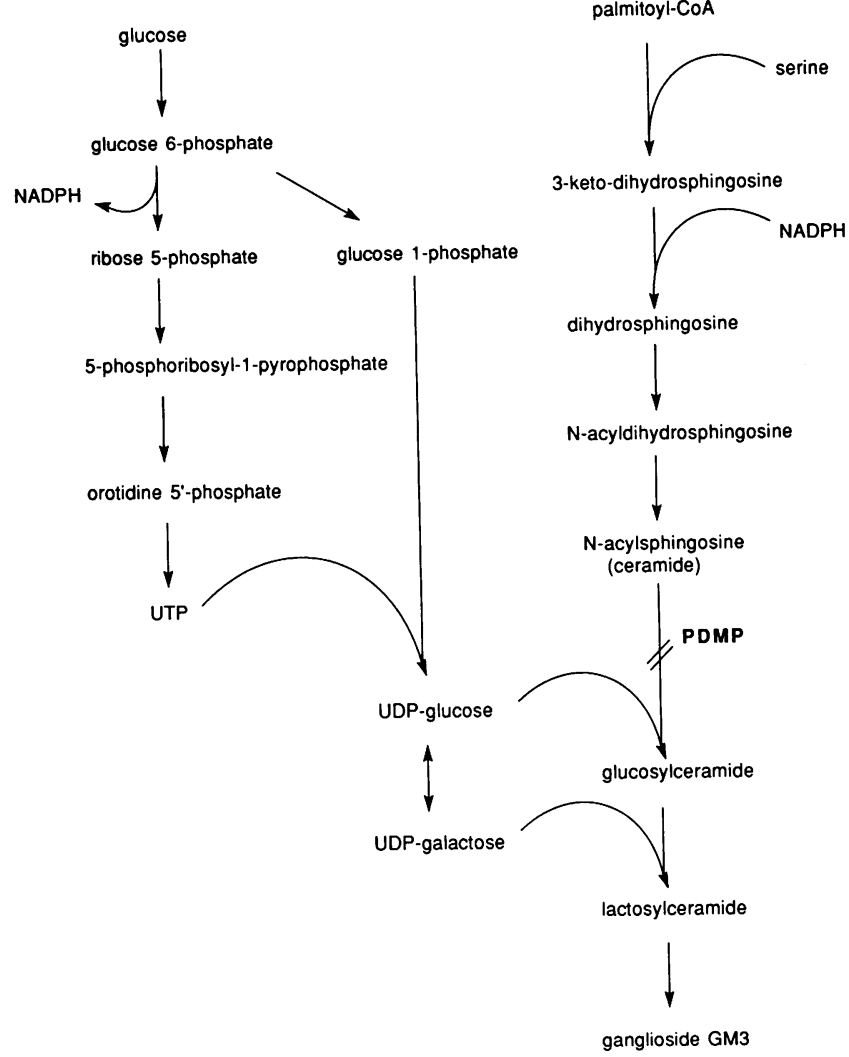

Figure 5. The pentose phosphate and sphingolipid synthetic pathways. Points of interaction between the pentose phosphate and glycosphingolipid pathways are shown. These include the formation of NADPH, a cofactor in dihydrosphingosine synthesis, and UDP-glucose and UDP-galactose, precursors for glucosyl- and lactosylceramide. PDMP blocks the formation of glucosylceramide through inhibition of the synthase which uses ceramide and UDP-glucose.

trol kidneys $\left(42.0 \times 10^{4} \pm 2.02 \times 10^{4}\right.$ versus $32.8 \times 10^{4} \pm 1.13$ $\left.\times 10^{4} \mu \mathrm{m}^{3}\right)$. PDMP treatment of the nondiabetic rats had no effect on glomerular size $\left(34.6 \times 10^{4} \pm 10.8 \times 10^{4} \mu \mathrm{m}^{3}\right)$. However, the glomerular volumes of diabetic animals treated with the glucosylceramide synthase inhibitor were not significantly different from those of the control groups $\left(34.8 \times 10^{4} \pm 9.23\right.$ $\left.\times 10^{3} \mu \mathrm{m}^{3}\right)$.

\section{Discussion}

Glycosphingolipid synthesis is dependent on two products of the pentose phosphate pathway (Fig. 5). NADPH is a required cofactor for the synthesis of both dihydrosphingosine and fatty acids, precursors for ceramide. UTP is the precursor for uridine diphosphohexoses, substrates for glycosyltransferases required in the formation of glucosylceramide and lactosylceramide. In the present study we considered the possibility that the metabolism of glucose through the pentose phosphate pathway results in higher levels of renal glycosphinglipids under hyperglycemic conditions.

Figure 3. Renal histology from control and diabetic groups. Hematoxylin and eosin-stained sections of kidney were obtained from control $(A)$; diabetic $(B)$; PDMP-treated $(C)$; and diabetic, PDMP-treated animals $(D)$. 
Sphingolipids have been implicated in a variety of important cellular functions, including growth, bacterial adhesion, and hormonal signaling (reviewed in [20]). These lipids, however, have not been systematically evaluated in models of diabetic nephropathy. We report that male rats rendered diabetic with streptozotocin accumulated GlcCer and ganglioside GM3 in their kidneys. These changes appear to be the result of increased substrate availability since the level of UDP-Glc, a precursor for glucosphingolipid synthesis, was increased in the rats with high blood glucose levels.

Cortes (4) and Spiro (5) and their co-workers have reported an increase in uridine diphosphohexoses in rat kidneys after experimentally induced diabetes. In particular, levels of UDP-Glc were demonstrated to be consistently increased in early diabetes. This increase appears to reflect enhanced synthesis of ribose-containing nucleotides and is supported by several observations. The kidney glucose concentration is elevated in diabetic kidney as are its reaction products, glucose-6-phosphate and UDP-Glc (21). Studies in diabetic rat kidney, using specifically labeled glucose, have identified increased flux through the pentose phosphate pathway in the absence of comparable changes in the tricarboxylic acid cycle (28). In addition, the activities of glucose-6-phosphate dehydrogenase and 6-phosphogluconate dehydrogenase are increased in diabetic kidney (29) as are the levels of UTP and orotate incorporation into UTP (22).

Since the observed concentration of UDP-Glc in normal kidney is $\sim 150 \mu \mathrm{M}$ and the $K_{\mathrm{m}}$ for this substrate is $250 \mu \mathrm{M}$, it appears that the glucosyltransferase is far from saturated with the substrate and the rate of synthesis would be proportional to the UDP-Glc concentration. Caution, of course, is required in drawing conclusions based on in vitro measurements of enzyme kinetics and estimated whole tissue concentrations of substrate. Some nucleotide sugars may be highly compartmentalized and present at significantly higher concentrations due to active transport processes at the site of the glycosyltransferase in question. This does not, however, appear to be the case for UDP-Glc. GlcCer synthase is situated on the outer leaflet of the Golgi and therefore is presumably subject to the cytosolic concentrations of UDP-Glc $(18,19)$. Because $>94 \%$ of the renal UDP-Glc is present in the cytosolic compartment, the measured tissue levels of UDP-Glc probably reflect the concentration of substrate available to the synthase.

The ceramide concentration in the region of GlcCer synthase may also be a factor. Ceramide levels may be regulated by glucose metabolites. These include NADPH, a cofactor for both dihydrosphinganine formation and fatty acid biosynthesis; acetyl-CoA, a substrate for fatty acid biosynthesis, or diacylglycerol, a precursor for ceramide formation via phosphorylcholine exchange between lecithin and sphingomyelin. Other inhibitors or activators of the enzyme may, of course, be present and diluted or eliminated under the assay conditions. Gangliosides have recently been demonstrated to inhibit GlcCer synthesis (23). Clearly, significant questions remain regarding the properties of the GlcCer synthase and its regulation in diabetic kidney.

The observed increase in renal GSL content in early streptozotocin-induced diabetes is potentially important since these glycoconjugates have been shown to regulate a variety of important cellular functions. Cellular growth and proliferation are regulated by changes in endogenous levels of specific GSLs and by the addition of exogenous GSLs. This is particularly relevant because renal hypertrophy, and to a lesser extent hyperplasia, are hallmarks of early diabetic nephropathy. Recently, proliferative changes in a renal epithelial cell line were described in association with altered GlcCer content (24). Increasing GlcCer content by inhibition of GlcCer $\beta$-glucosidase stimulated epithelial cell growth; decreasing GlcCer by inhibition of GlcCer synthase resulted in impaired proliferation. In addition, testosterone-induced renal hypertrophy was associated with increased activity of GlcCer synthase and decreased hydrolase activity (25). Castration of mice with alloxan-induced diabetes decreases but does not reverse renal hypertrophy (26). Exposure of mice to a GlcCer synthase inhibitor resulted in the rapid loss of GlcCer, consistent with a very high turnover of this sphingolipid in murine kidney (11). Lactosylceramide has been recently reported to exhibit growth stimulatory effects when added to cultured cells $(27,28)$. These growth-related changes may be the result of alteration in extracellular matrix binding, modulation of tyrosine kinase activity, or changes in protein kinase $\mathrm{C}$. Protein kinase $\mathrm{C}$ activity may be altered due to changes in free sphingoid bases such as sphingosine, dihydrosphingosine, or glucosylsphingosine (29), which are products of sphingolipid metabolism, or due to the generation of diglyceride as a byproduct of sphingomyelin synthesis (30).

Glycosphingolipids have been demonstrated to modulate other signaling systems as well. Recently, GlcCer has been reported to play a potential regulatory role in phospholipase Cgenerated inositol trisphosphate $(31,32)$. Ganglioside GM3 and its metabolites have been demonstrated to exert significant effects on tyrosine kinase activity (33). Signaling derangements have been suggested to be present in diabetic tissues, particularly in the coupling of angiotensin II to phospholipase C (34). The potential role of altered GSL expression in modulating these changes will require further investigation.

Glycosphingolipids have also been identified as receptors for bacterial adhesins and toxins in many tissues, including the urinary tract (35). An increased expression of GSLs may contribute to the known increased susceptibility of diabetics to bacterial infection.

In conclusion, streptozotocin-induced diabetes results in an increase in GSL content in the kidneys of male rats. These findings indicate the importance of a quantitatively minor route for glucose utilization in hyperglycemic states. The increase in GSLs parallels the increase in UDP-Glc and is prevented by concurrent insulin administration. Moreover, PDMP administration reverses the early hypertrophy of insulin deficient diabetes mellitus. The ability to alter renal growth by inhibition of GSL synthesis may provide a new pharmacological approach to the treatment of diabetic complications. The possibility that diabetic nephropathy may represent an "acquired sphingolipidosis" and that GSLs may mediate in part the pathophysiological consequences of diabetes in the kidney and other susceptible tissues merits further investigation.

\section{Acknowledgments}

This work was supported by National Institutes of Health grants RO1 DK41487 (J. A. Shayman) and a Merit Review Research Award from the Veterans Administration (J. A. Shayman). N. S. Radin is a Jacob Javits Investigator Awardee. 


\section{References}

1. Beyer-Mears, A., L. Ku, and M. P. Cohen. 1984. Glomerular polyol accumulation in diabetes and its prevention by oral sorbinil. Diabetes. 33:604-607.

2. Brownlee, M. 1991. Glycosylation products as toxic mediators of diabetic complications. Annu. Rev. Med. 42:159-166.

3. Craven, P. A., C. M. Davidson, and F. R. DeRubertis. 1990. Increase in diacylglycerol mass in isolated glomeruli by glucose from de novo synthesis of glycerolipids. Diabetes. 39:667-674.

4. Cortes, P., F. Dumler, K. S. Sastry, C. P. Verghese, and N. W. Levin. 1982. Effects of early diabetes on uridine diphosphosugar synthesis in the rat renal cortex. Kidney Int. 21:676-682.

5. Spiro, M. J. 1984. Effect of diabetes on the sugar nucleotides in several tissues of the rat. Diabetologia. 26:70-75.

6. Radin, N. S. 1976. Preparative isolation of cerebrosides (galactosyl and glucosyl ceramide). J. Lipid. Res. 17:290-293.

7. Hirose, K., R. Osterby, M. Nozawa, and H. J. G. Gundersen. 1982. Development of glomerular lesions in experimental long-term diabetes in the rat. Kidney Int. 21:689-695.

8. Steer, K. A., M. Sochor, A. M. Gonzalez, and P. McLean. 1982. Regulation of pathways of glucose metabolism in kidney. Specific linking of pentose phosphate pathway activity with kidney growth in experimental diabetes and unilateral nephrectomy. FEBS (Fed. Eur. Biochem. Soc.) Lett. 150:494-498.

9. Steer, K. A., M. Sochor, and P. McLean. 1985. Renal hypertrophy in experimental diabetes. Changes in pentose phosphate pathway activity. Diabetes. $34: 485-490$

10. Touchstone, J. C., S. S. Levin, M. F. Dobbins, L. Matthews, P. C. Beers, and S. G. Gabbe. 1983. (3-sn-Phosphatidyl)cholines (lecithins) in amniotic fluid. Clin. Chem. 29:1951-1954.

11. Shukla, G. S., A. Shukla, J. Inokuchi, and N. S. Radin. 1991. Rapid kidney changes resulting from glycosphingolipid depletion by treatment with a glucosyltransferase inhibitor. Biochim. Biophys. Acta. 1083:101-108.

12. Keppler, D., J. Rudigier, and K. Decker. 1970. Enzymic determination of uracil nucleotides in tissues. Anal. Biochem. 38:105-114.

13. Dinur, T., G. A. Grabowski, R. J. Desnick, and S. Gatt. 1984. Synthesis of a fluorescent derivative of glucosyl ceramide for the sensitive determination of glucocerebrosidase activity. Anal. Biochem. 136:223-234.

14. Shukla, G. S., and N. S. Radin. 1990. Glucosyceramide synthase of mouse kidney: further characterization with an improved assay method. Arch. Biochem. Biophys. 283:372-378.

15. Shukla, A., and N. S. Radin. 1991. Metabolism of D- $\left[{ }^{3} \mathrm{H}\right]$ threo-1-phenyl2-decanoylamino-3-morpholino-1-propanol, an inhibitor of glucosylceramide synthesis, and the synergistic action of an inhibitor of microsomal monooxygenase. J. Lipid Res. 32:713-722.

16. Weibel, E. R. 1979. Stereological Methods Practical Methods for Biological Morphometry. Academic Press, Ltd., London. 44-45.

17. Weinberg, J. M., P. G. Harding, and H. D. Humes. 1983. Alterations in renal cortex cation homeostasis during mercuric chloride and gentamicin nephrotoxicity. Exp. Mol. Pathol. 39:43-60.
18. Coste, H. M. B. Martel, and R. Got. 1986. Topology of glucosylceramide synthesis in Golgi membranes from porcine submaxillary glands. Biochim. Biophys. Acta. 858:6-12.

19. Futerman, A. H., and R. E. Pagano. 1991. Determination of the intracellular sites and topology of glucosylceramide synthesis in rat liver. Biochem. $J$. 280:295-302.

20. Shayman, J. A., and N. S. Radin. 1991. Structure and function of renal glycosphingolipids. Editorial. Am. J. Physiol. 260:F291-F302.

21. Needleman, P., J. V. Passonneau, and O. H. Lowry. 1968. Distribution of glucose and related metabolites in rat kidney. Am. J. Physiol. 215:655-659.

22. Cortes, P., F. Dumler, and N. W. Levin. 1988. Glomerular uracil nucleotide synthesis. Am. J. Physiol. 255:F635-F646.

23. Shukla, G., A. Shukla, and N. S. Radin. 1991. Gangliosides inhibit glucosylceramide synthase: possible role in ganglioside therapy. J. Neurochem. $56: 2125-2132$

24. Shayman, J. A., G. D. Deshmukh, S. Mahdiyoun, T. P. Thomas, D. Wu, F. S. Barcelon, and N. S. Radin. 1991. Modulation of renal epithelial cell growth by glucosylceramide: association with protein kinase $\mathrm{C}$, sphingosine, and diacylglycerol. J. Biol. Chem. 266:22968-22974.

25. Shukla, A., G. S. Shukla, and N. S. Radin. 1992. Control of kidney size by sex hormones: possible involvement of glucosylceramide. Am. J. Physiol 262:F24-F29.

26. Broulik, P. D., and V. Schreiber. 1982. Effect of alloxan diabetes on kidney growth in intact and castrated mice. Acta Endocrinol. 99:109-111.

27. Chatterjee, S. 1991. Lactosylceramide stimulates aortic smooth muscle cell proliferation. Biochem. Biophys. Res. Commun. 181:554-561.

28. Ogura, K., and C. C. Sweeley. 1991. Mitogenic response of cells treated with bacterial sialidase and lactosylceramide. Glycoconjugate J. 8:160. (Abstr.)

29. Hannun, Y. A., and R. M. Bell. 1989. Functions of sphingolipids and sphingolipid breakdown products in cellular regulation (comments). Science (Wash. DC). 243:500-507.

30. Merrill, A. H., Jr., and D. D. Jones. 1990. An update of the enzymology and regulation of sphingomyelin metabolism. Biochim. Biophys. Acta. 1044:112.

31. Shayman, J. A., S. Mahdiyoun, G. Deshmukh, F. Barcelon, J. Inokuchi, and N. S. Radin. 1990. Glucosphingolipid dependence of hormone-stimulated inositol trisphosphate formation. J. Biol. Chem. 265:12135-12138.

32. Mahdiyoun, S., A. Abe, G. D. Deshmukh, N. S. Radin, and J. A. Shayman. 1992. Decreased formation of inositol trisphosphate in Madin-Darby canine kidney cells under conditions of $\beta$-glucosidase inhibition. Arch. Biochem. Biophys. 292:506-511.

33. Bremer, E. G., J. Schlessinger, and S. Hakomori. 1986. Ganglioside-mediated modulation of cell growth. Specific effects of GM 3 on tyrosine phosphorylation of the epidermal growth factor receptor. J. Biol. Chem. 261:2434-2440.

34. Zatz, R., and B. M. Brenner. 1986. Pathogenesis of diabetic microangiopathy. The hemodynamic view. Am. J. Med. 80:443-453.

35. Karlsson, K. A. 1989. Animal glycosphingolipids as membrane attachment sites for bacteria. Annu. Rev. Biochem. 58:309-350. 